\title{
Geschäftsbericht 2014 der FMH Services Genossenschaft
}

Die FMH Services Genossenschaft darf auf ein erfolgreiches Geschäftsjahr 2014 zurückblicken und konnte in allen Geschäftsbereichen ihre Aktivitäten und Kundenbeziehungen ausbauen. Die Finanzanlagen, die mit einer auf Sicherheit basierenden Strategie bewirtschaftet werden, erzielten erneut eine erfreuliche Rendite. Der Geschäftsbericht 2014 kann auf unserer Website (www.fmhservices.ch) abgerufen werden.

Die FMH Services erbringen seit 90 Jahren in enger Partnerschaft mit der Verbindung der Schweizer Ärztinnen und Ärzte FMH betriebswirtschaftliche Dienstleistungen. Der gültige Zusammenarbeitsvertrag wird derzeit im Rahmen einer Gesamtstrategie diskutiert und aktualisiert. Ziel ist die Verfestigung der Zusammenarbeit zum Nutzen der Mitglieder bzw. Genossenschafter.
Dieses Jahr werden Wahlen für die Verwaltung der FMH Services durchgeführt. Dr. rer. publ. HSG Sven Bradke, Dr. med. Vincenzo Liguori und Dr. med. Pierre Sindelar stellen sich der Wiederwahl. Infolge meines statutarisch bedingten Rücktritts wird die Wahl eines neuen Präsidenten notwendig. Dr. Beat Bumbacher, der 2014 als neues Mitglied der Verwaltung zu uns gestossen ist, hat sich erfreulicherweise bereit erklärt, ab Ende 2015 dieses Amt zu übernehmen.

Ich danke der Ärzteschaft für das uns entgegengebrachte Vertrauen und die anregenden Kundenbeziehungen. Geschäftspartnern und Mitarbeitenden danke ich für das grosse Engagement, das sie der Dienstleistungsorganisation der Schweizer Ärztinnen und Ärzte entgegengebracht haben.
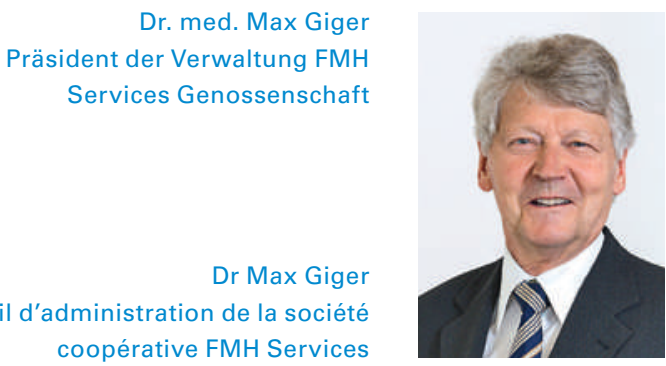

\section{Rapport de gestion 2014 de la coopérative FMH Services}

L'exercice 2014 de la société coopérative FMH Services a été couronné de succès. Nous avons pu étendre nos activités et renforcer les liens avec nos clients dans tous nos domaines d'activité. Notre stratégie financière, basée sur des investissements sûrs, a permis de dégager des rendements intéressants.

Le rapport de gestion 2014 peut être téléchargé de notre site web (www.fmhservices.ch).

Cela fait 90 ans que FMH Services propose des prestations en matière d'économie d'entreprise en partenariat avec la Fédération des médecins suisses. La convention en vigueur qui lie les deux entités est actuellement discutée et remaniée dans le cadre de la stratégie globale afin de renforcer encore notre collaboration pour le bien des membres et sociétaires.

Cette année, il y aura de nouvelles élections au conseil d'administration. Sven Bradke, Dr rer. publ. HSG, le Dr Vincenzo Liguori et le Dr Pierre Sindelar se portent candidats à leur réélection. En raison de ma démission pour raisons statutaires, la nomination d'un nouveau président s'imposait et c'est le Dr Beat Bumbacher, membre du conseil d'administration depuis 2014, qui s'est proposé d'assumer cette tâche dès la fin 2015.

Je remercie cordialement le corps médical de sa confiance ainsi que nos partenaires et nos collaborateurs pour le grand engagement dont ils font preuve au sein de la société de services des médecins suisses. 